\title{
Celebrating 75 years of the powder diffraction File ${ }^{\mathrm{TM}}$
}

Soorya N Kabekkodu ${ }^{1}$, Justin Blanton ${ }^{2}$, Thomas Blanton ${ }^{3}$

${ }^{1}$ Database Deparatment, International Centre For Diffraction Data, Newtown Square, United States, ${ }^{2}$ Engineering \&Design International Centre for Diffraction Data, Newtown Square, PA, United States, ${ }^{3}$ Executive Director, International Centre for Diffraction Data, Newtown Square, PA, United States

E-mail: kabekkodu@icdd.com

The International Centre for Diffraction Data (ICDD®) Powder Diffraction File (PDF®) is a powerful database for materials characterization and has been used extensively by the scientific community for 75 years. Over this period, the database has grown exponentially, housing more than 890,000 diffraction patterns and 330,000 crystal structures in Release 2016 . The Powder Diffraction File has a wealth of information that a materials scientist can take advantage of in various ways, from materials identification, characterization, computation to design. Starting with 1,000 hand written file cards in 1941 , the Powder Diffraction File has undergone numerous technological developments in the database as well as search/match and data retrieval methods. Today, the Powder Diffraction File in Relational Database Format (RDB) format contains extensive chemical, physical, bibliographic and crystallographic data including atomic coordinates enabling characterization and computational analysis. While using any crystallographic database, it is important to know the quality of the crystal structure or diffraction pattern found in the database. With varying quality of the published data in the literature, ICDD database editorial review processes had to adopt rigorous data evaluation and computational methods to classify data based on its quality. Every entry in the Powder Diffraction File has a quality mark and editorial comments describing original data errors and any corrections. In addition to X-ray diffraction patterns, the PDF also contains electron and neutron diffraction patterns enabling scientists to use different diffraction techniques to characterize the material of interest. Recently, ICDD extended the scope of the database to include modulated structures. Modulated structures are of interest due to their technologically important properties such as, ferroelectricity, luminescence, superconductivity and thermoelectricity. As these structures do not have lattice periodicity in regular three dimension space, they need to be described in $3+d$ dimension (super space approach). This requires different computational algorithms for database implementation and data retrieval.

Various computational methods and search/match algorithms implemented in the Powder Diffraction File that are of interest in computational materials science and characterization will be discussed in this presentation. This presentation will also emphasize the database-assisted analysis of disordered clays, polymers, amorphous materials and nanomaterials.

Keywords: Crystalllographic Databases; Phase Identification; Powder Diffraction 